\title{
EFEITO BIOCIDA DE MANDELATOS ORGANOESTÂNICOS SOBRE Fusarium oxysporum $f$. sp. cubense ${ }^{1}$
}

\author{
Biocide effect of organotin mandelates on Fusarium oxysporum $f$. sp. cubense \\ Edelcio Tavares de Araujo ${ }^{2}$, Walclée de Carvalho Melo ${ }^{3}$, \\ Mário César Guerreiro ${ }^{3}$, Roberto Santos Barbiéri ${ }^{4}$, Celeste Maria Patto de Abreu
}

\begin{abstract}
RESUMO
Compostos organoestânicos são substâncias que têm sido utilizadas como biocidas. A vantagem do uso dessa classe de compostos é sua degradação em dióxido de estanho $\left(\mathrm{SnO}_{2}\right)$, que é atóxico para o meio ambiente. Os complexos organoestânicos foram obtidos pela reação de quantidades eqüimolares dos isômeros dos ácidos R-(-)- e S-(+)-mandélicos e de dicloreto de difenilestanho, com o objetivo de testar o efeito biocida dos novos complexos sobre culturas do fungo Fusarium oxysporum f.sp. cubense, avaliando o potencial de inibição do crescimento micelial médio do fungo em teste direto in vitro, em meio de cultura $\mathrm{BDA}$, a $25^{\circ} \mathrm{C}$. Foram utilizadas as concentrações $0,25,100,250$ e $500 \mathrm{mg} / \mathrm{kg}$, em quadruplicatas, com avaliações em 2 , 4 e 7 dias. Os complexos R e S são insolúveis no meio BDA e os grânulos caoticamente dispersos efetivamente inibiram o crescimento fúngico.
\end{abstract}

Termos para indexação: Complexos organoestânicos, efeito biocida, fungo.

\begin{abstract}
Organotin compounds are substances which have been utilized as biocides. The advantage of using this class of compounds is their degradation into tin dioxide $\left(\mathrm{SnO}_{2}\right)$, which is non-toxic to environment. The organotin complexes were obtained from the reaction of equimolar amounts of the isomers of the acids $\mathrm{R}$ and $\mathrm{S}$ mandelic and diphenyltin dichloride with the purpose of testing the biocide effect of the new complexes on cultures of the fungus Fusarium oxysporum $f . s p$. cubense by evaluating in in vitro direct test in BDA culture medium at $25^{\circ} \mathrm{C}$. Concentrations of $0,25,100,250$ and $500 \mathrm{mg} / \mathrm{kg}$ were tested in quadruplicates, with readings after 2, 4 and 7 days. The $\mathrm{R}$ and $\mathrm{S}$ complexes are insoluble in the BDA medium, the chaotically dispersed grains effectively inhibited the fungal growth.
\end{abstract}

Index terms: Organotin complexes, biocide effect, fungus.

(Recebido para publicação em 10 de maio de 2002 e aprovado em 29 de julho de 2002)

\section{INTRODUÇÃO}

Por meio de investigações sistemáticas, a partir de 1950, vem-se conhecendo a ação biocida dos compostos organoestânicos que atuam sobre fungos e bactérias, vermes parasitários, insetos, caracóis e organismos marinhos (KERK, 1954, 1962). Compostos foram desenvolvidos com ação fitossanitária (fungicidas, antibacterianos, antihelmínticos, inseticidas, repelentes e biocidas em geral), com aplicação em várias áreas, como agricultura, veterinária, farmacologia e medicina (LUIGTEN, 1972; KUMARI et al., 1994; BONNETT, 1995). A vantagem do uso de compostos organoestânicos, biocidas de elevada toxidez a microorganismos, se dá por sua degradação, geralmente, em dióxido de estanho $\left(\mathrm{SnO}_{2}\right)$, atóxico para o meio ambiente.
Novas classes de compostos $\alpha$-hidroxicarboxilatos organoestânicos (PETTINARI et al., 2001; MATSUGI et al., 2001) têm sido investigados por Barbiére et al. (1989, 1994) Dias (1999) verificou que o mandelato de difenilestanho $\left[\left(\mathrm{C}_{6} \mathrm{H}_{5}\right)_{2} \mathrm{SnMand}_{2}\right]$ inibiu o crescimento micelial do fungo Fusarium oxysporum $f$. sp. cubense, [Mand $=\mathrm{C}_{6} \mathrm{H}_{5} \mathrm{CH}(\mathrm{OH}) \mathrm{COO}^{-}$].

A ação de fungos patogênicos tem causado grandes prejuízos e perdas irreparáveis na agricultura. Em 1920, na região de Piracicaba-SP, foi identificada a doença murcha-da-bananeira, causada pelo fungo Fusarium oxysporum $f$. sp. cubense, que provocou, em todo o Estado de São Paulo, a erradicação das variedades banana-maçã e banana-terra, variedades essas susceptíveis ao fungo, sendo substituídas

\footnotetext{
1. Extraído da dissertação de mestrado do primeiro autor.

2. Mestrando em Agroquímica e Agrobioquímica - Universidade Federal de Lavras/UFLA - Caixa Postal 37 - 37200-000 - Lavras, MG. Professor do Instituto Adventista de Ensino, Caixa Postal 144 - 37200-000 - Lavras, MG.

3. Químico, DsC., Departamento de Química/UFLA.

4. Químico, DsC., Faculdade Ubaense Ozanam Coelho/FAGOC - 36500-000 - Ubá, MG

5. Engenheira Agrônoma, DsC, Departamento de Química/UFLA.
} 
pelas banana nanica e nanicão, variedades resistente ao patógeno.

Revestem-se de importância a pesquisa e a identificação de produtos e substâncias que exercem ação biocida sobre fungos patogênicos. Com este trabalho, objetivou-se sintetizar os compostos organoestânicos a partir dos isômeros R-(-)- e S-(+)- do ácido mandélico, em reação com o dicloreto de difenilestanho e testar a ação inibitória do crescimento do fungo.

\section{MATERIAL E MÉTODOS}

Foram realizadas as sínteses dos complexos organoestânicos R e S, espectroscopia de infravermelho, análise de ponto de fusão, análise elementar de C e $\mathrm{H}$, bem como testes da atividade inibitória do crescimento fungico sob a ação dos reagentes dicloreto de difenilestanho, dos ácidos R-(-)- e S-(+)-mandélicos e ação dos complexos R e S, em culturas do fungo $F u$ sarium oysporum $f$. sp. cubense. Os inóculos para a realização dos testes com o fungo foram obtidos de culturas-padrão, mantidos em meio BDA (batata, dextrose e agar) a $25^{\circ} \mathrm{C}$, sendo repicados e aplicados após decorridos sete dias. Foram utilizadas concentrações de $0,25,100,250$ e $500 \mathrm{mg} / \mathrm{kg}$, em quadruplicatas, com medidas do diâmetro micelial do crescimento fungíco em 2, 4 e 7 dias, sendo feitas posteriormente as análises estatísticas dos dados de crescimentodo fungo.

\section{Síntese dos complexos organoestânicos $\mathrm{R}$ e $\mathrm{S}$}

Em um balão de fundo redondo de $50 \mathrm{~mL}$, foi dissolvido $0,250 \mathrm{~g}(1,65 \mathrm{mmol})$ de ácido R-(-)mandélico em $20 \mathrm{~mL}$ de acetonitrila e adicionado 0,550 $\mathrm{g}(1,60 \mathrm{mmol})$ de dicloreto de difenilestanho. O sistema foi mantido sob agitação magnética e refluxo a $80^{\circ} \mathrm{C}$ por 24 horas. $\mathrm{O}$ volume da mistura foi reduzido até a metade do volume inicial, em evaporador rotatório. Deixouse a mistura em repouso, sob refrigeração na temperatura de $-18^{\circ} \mathrm{C}$, até a formação de um precipitado de aparência cristalina. $\mathrm{O}$ precipitado, complexo $\mathrm{R}$, que se formou após 74 dias, foi separado por filtração em funil com placa de vidro sinterizado número 4 e lavado com três porções de $2 \mathrm{~mL}$ de acetonitrila, sendo obtido 0,212 $\mathrm{g}$, correspondendo a $28,8 \%$ de rendimento. O sólido obtido foi transferido para um dessecador e mantido sob vácuo, (TERRA, 1997; TERRA et al., 1998). Utilizouse o ácido S-(+)-mandélico como reagente e o mesmo procedimento para obtenção do complexo S. Sua precipitação ocorreu após 62 dias, sendo obtido $0,352 \mathrm{~g}$, correspondendo a $47,9 \%$ de rendimento.

Os espectros de infra vermelho foram obtidos em um espectrofotômetro Shimadzu FTIR-8201 A, com transformada de Fourier, (4600 a $500 \mathrm{~cm}^{-1}$ ), usando a técnica de pastilhamento com brometo de potássio (K$\mathrm{Br}$ ). As análises de carbono e hidrogênio foram realizadas em um microanalizador CHN, modelo 2400 da Perkin-Elmer; na determinação de ponto de fusão aparente, foi utilizado o equipamento Bücchi, modelo 535.

\section{RESULTADOS E DISCUSSÃO}

\section{Espectroscopia de absorção de infravermelho}

Os espectros do dicloreto de difenilestanho, dos ácidos R-(-)- e S-(+)-mandélicos e dos complexos $\mathrm{R}$ e $\mathrm{S}$ estão apresentados na FIGURA 1 e na TABELA 1. Nos complexos $R$ e $S$ houve um deslocamento da


$1.724 \mathrm{~cm}^{-1}$ referente aos ácidos R-(-)- e S-(+)mandélicos, para regiões de frequiências mais baixas, 1.560-1.562 $\mathrm{cm}^{-1}$. Os ácidos R-(-)- e S-(+)mandélicos apresentam uma banda fina característica da freqüência de estiramento $v_{\mathrm{OH} \text { (alcoólica), na região }}$ de $3.448 \mathrm{~cm}^{-1}$. Com a interação do metal e o átomo de oxigênio da hidroxila alcoólica, essa banda apresenta-se larga. Isso indica formação do quelato por meio da coordenação tanto do grupo hidroxila quanto do grupo carboxilato do ácido mandélico.

\section{Analise elementar de carbono e hidrogênio e determinação de ponto de fusão aparente}

Os resultados analíticos de carbono e hidrogênio estão apresentados na TABELA 2. Os percentuais experimentais, em relação aos valores teóricos calculados, apresentam pequenas diferenças que podem ser devidas às possíveis impurezas no composto isolado. As porcentagens de hidrogênio e carbono encontradas experimentalmente estão de acordo com a calculada.

Em temperatura ambiente, os complexos $\mathrm{R}$ e $\mathrm{S}$ são sólidos, na forma de pó com coloração branca. Os valores obtidos para os pontos de fusão ficaram comprometidos, pois a diferença de temperatura entre esse evento e a decomposição dos compostos deve ser pequena, passando esses a apresentar coloração cinza. 




FIGURA 1 - Espectros de absorção na região do infravermelho: (a) dicloreto difenilestanho, (b) ácido R-(-)mandélico e (c) complexo R. 
TABELA 1 - Dados $\left(\mathrm{cm}^{-1}\right)$ dos espectros de absorção na região do infravermelho do dicloreto de difenilestanho, ácidos R-(-)- e S-(+)-mandélico e dos complexos S e R.

\begin{tabular}{|c|c|c|c|c|}
\hline Atribuições & $v_{\mathrm{C}-\mathrm{H}(\text { arom.) }}$ & $v_{\mathrm{C}=\mathrm{C}(\text { arom.) }}$ & $v_{\mathrm{OH}}$ & $v_{\mathrm{COOH} \text { (assim.) }}$ \\
\hline \multirow{2}{*}{ Dicloreto de difenilestanho } & 3.068 & 1.480 & & \\
\hline & 3.056 & 1.433 & & \\
\hline Ácido R-(-)-mandélico & & & 3.448 & 1.724 \\
\hline Ácido S-(+)-mandélico & & & 3.448 & 1.724 \\
\hline \multirow{2}{*}{ Complexo-R } & 3.058 & 1.481 & 3.290 & 1.560 \\
\hline & & 1.433 & & \\
\hline \multirow{2}{*}{ Composto-S } & - & 1.481 & 3.350 & 1.562 \\
\hline & & 1.434 & & \\
\hline
\end{tabular}

TABELA 2 - Resultados analíticos de C e H dos complexos R-(-) e S-(+)-mandélico.

\begin{tabular}{lccccc}
\hline \multirow{2}{*}{ Complexo } & \multirow{2}{*}{$\begin{array}{c}\text { Temperatura de } \\
\text { decomposição }\end{array}$} & Calc. & Exp. & Calc. & Exp. \\
\cline { 3 - 6 } & $180^{\circ} \mathrm{C}$ & 43,95 & 43,32 & 3,14 & 3,63 \\
\hline R-(-)-mandélico & $180^{\circ} \mathrm{C}$ & 43,95 & 44,53 & 3,14 & 3,75 \\
\hline
\end{tabular}

Calc. $=$ calculado, $\quad$ Exp. $=$ experimental,

\section{Testes de atividade biológica}

Os testes de inibição do crescimento do fungo Fusarium oxysporum f.sp.cubense foram realizados para cada um dos reagentes de partida: os ácidos R-(-)-, S(+)-mandélicos, o dicloreto de difenilestanho e para cada um dos compostos obtidos, os complexos R e S. Para efeito de comparação e análise, foram aplicados em culturas do fungo, in vitro, em meio BDA a $25^{\circ} \mathrm{C}$, nas concentrações de 0 (testemunha), 25, 100, 250, $500 \mathrm{mg} / \mathrm{kg}$, em quadruplicatas. $\mathrm{O}$ crescimento fúngico foi medido pelo diâmetro micelial, ao longo de dois eixos ortogonais entre si, considerando-se a média dos valores. Os compostos dicloreto de difenilestanho e os complexos $\mathrm{R}$ e $\mathrm{S}$ são insolúveis, apresentando grânulos com granu- lometria variada e caoticamente dispersos no meio de cultura BDA. Os dados foram tratados estatisticamente pelo Sistema de Análise Estatística - SANEST, (ZONTA e MACHADO, 1991).

Na FIGURA 2 estão representados os dados do crescimento micelial médio do Fusarium oxysporum f.sp. cubense sob ação do ácido S-(+)-mandélico, dicloreto de difenilestanho e dos complexos $\mathrm{R}$ e $\mathrm{S}$, e na TABELA 3, dados do ácido R-(-)-mandélico, para as concentrações $0,25,100,250$ e $500 \mathrm{mg} / \mathrm{kg}$. Verifica-se que todos os compostos aumentam o seu poder de inibição do crescimento fúngico com o aumento da concentração. 




FIGURA 2 - Crescimento médio micelial do fungo Fusarium oxyxporum $f$. sp. cubense, sob a ação de diferentes compostos e concentrações.

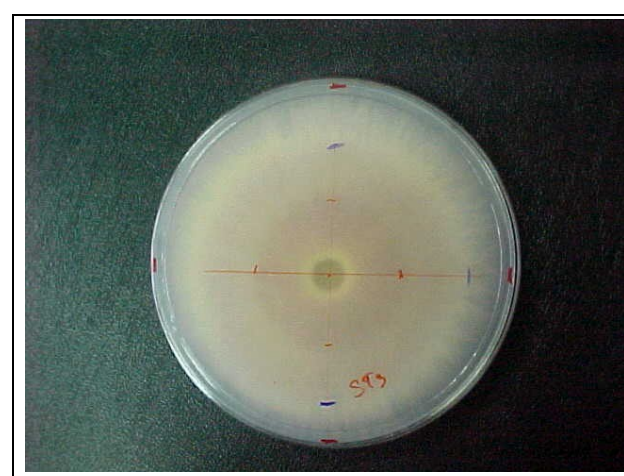

Testemunha



$100 \mathrm{mg} / \mathrm{kg}$

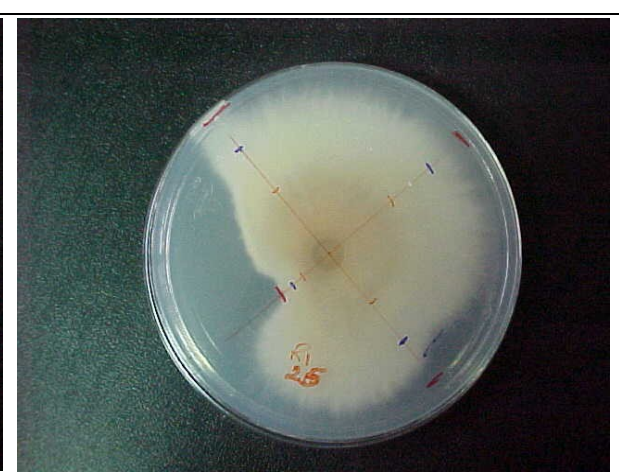

$25 \mathrm{mg} / \mathrm{kg}$

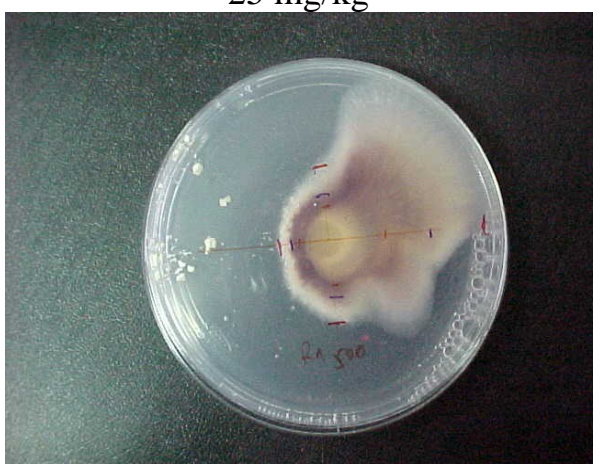

$500 \mathrm{mg} / \mathrm{kg}$

FIGURA 3 - Fotografias das placas de crescimento micelial do fungo Fusarium oxysporum f.sp.cubense, em meio BDA $(0,25,100$ e $500 \mathrm{mg} / \mathrm{kg})$, na presença do complexo R após um período de 7 dias. 
TABELA 3 - Crescimento médio micelial do fungo Fusarium oxysporum f.sp. cubense, sob ação do ácido Rmandélico, diâmetro médio para diferentes concentrações.

\begin{tabular}{cc}
\hline Concentração $(\mathbf{m g} / \mathbf{k g})$ & Diâmetro médio $\mathbf{( c m})$ \\
\hline 0 & 4,15 \\
25 & 4,51 \\
100 & 4,25 \\
250 & 4,22 \\
500 & 3,85 \\
\hline
\end{tabular}

Não significativo a $5 \%$ pelo teste $F$.

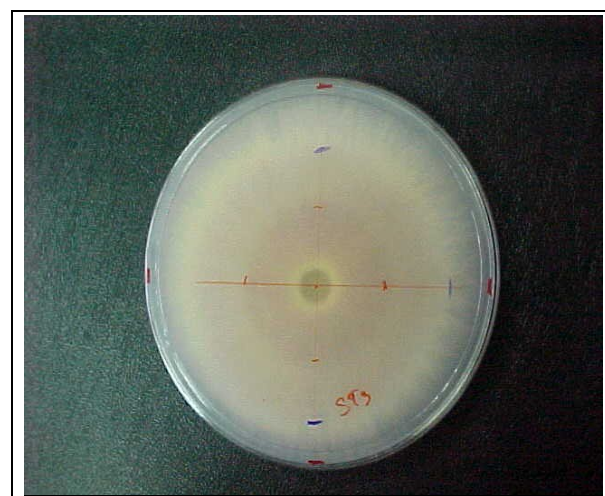

Testemunha

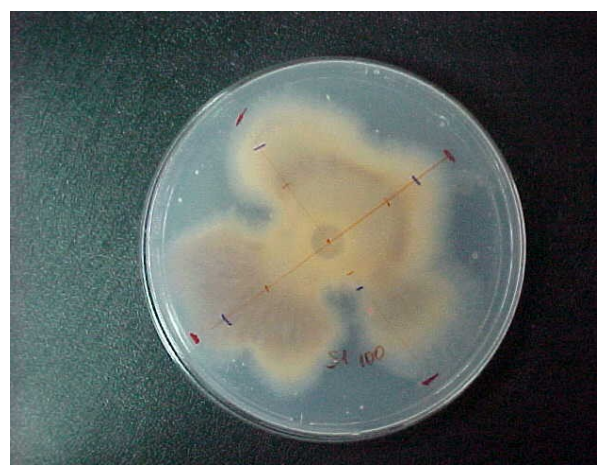

$100 \mathrm{mg} / \mathrm{kg}$

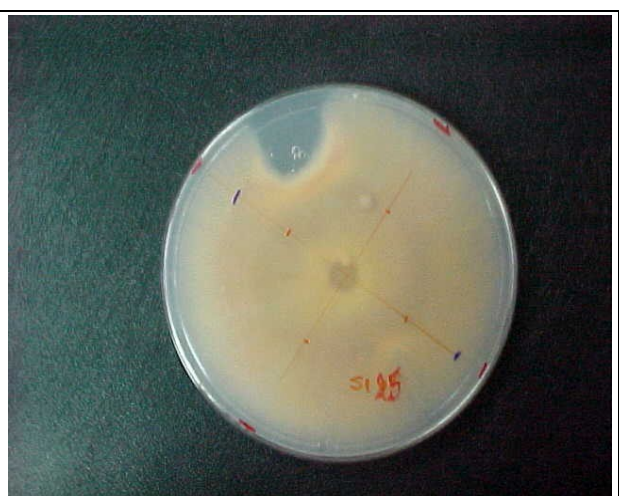

$25 \mathrm{mg} / \mathrm{kg}$

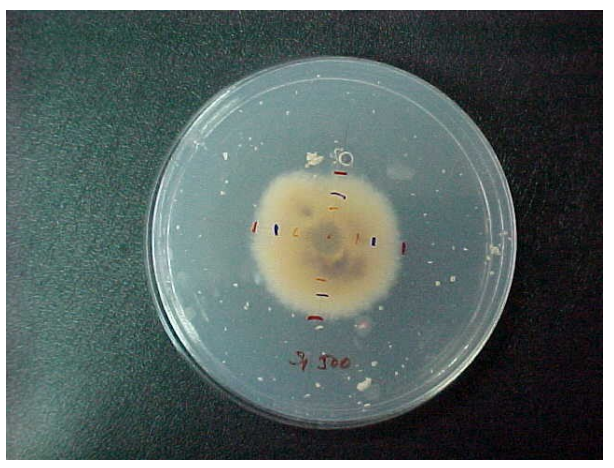

$500 \mathrm{mg} / \mathrm{kg}$

FIGURA 4 - Fotografias das placas de crescimento micelial do fungo Fusarium oxysporum f.sp.cubense, em meio BDA $(0,25,100$ e $500 \mathrm{mg} / \mathrm{kg})$, na presença do complexo $\mathrm{S}$ após um período de 7 dias. 




2 dias

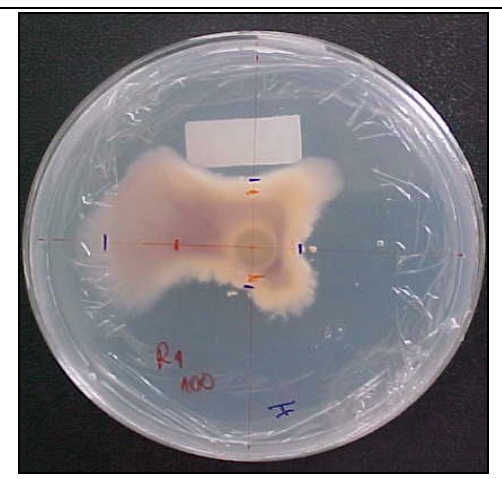

4 dias

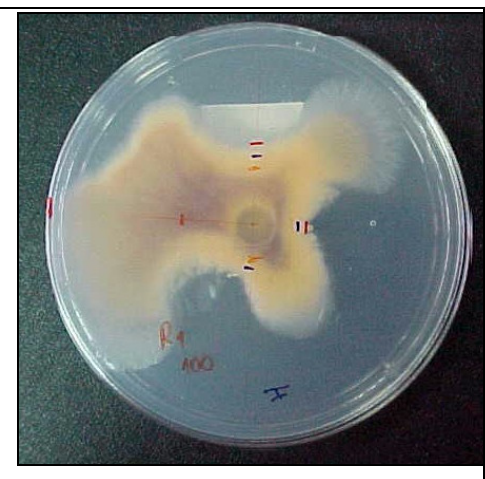

7 dias

FIGURA 5 - Fotografias das placas de crescimento micelial do fungo Fusarium oxysporum f.sp.cubense, em meio BDA, na concentração de $100 \mathrm{mg} / \mathrm{kg}$, na presença do complexo R, nos períodos de 2, 4 e 7 dias.

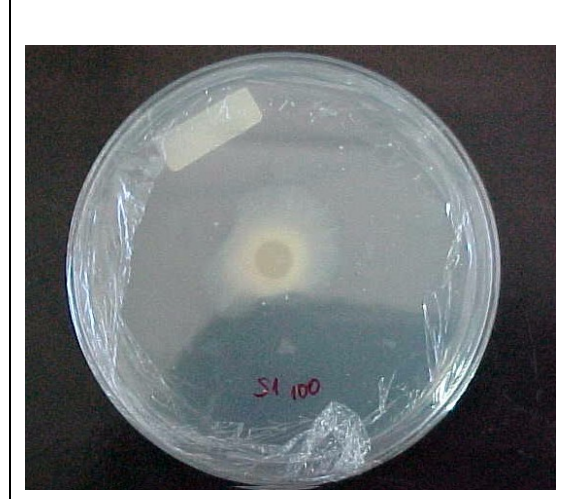

2 dias

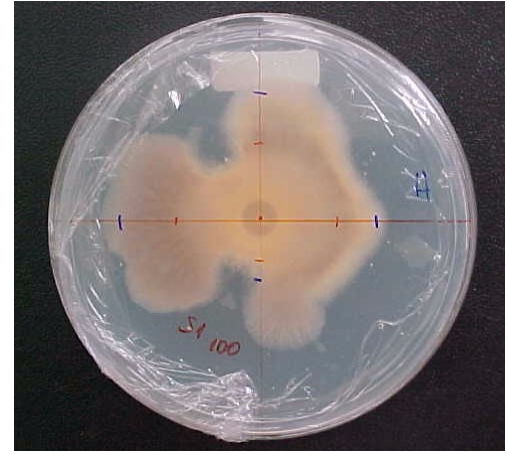

4 dias

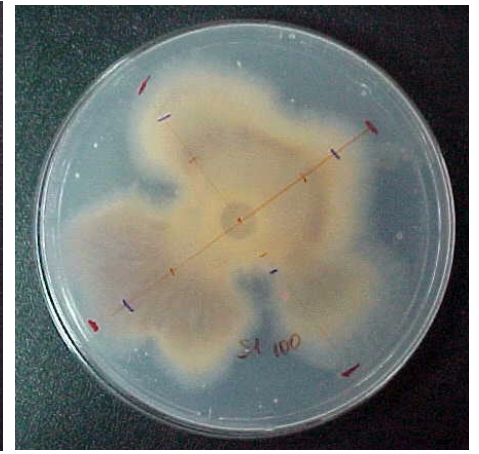

7 dias

FIGURA 6 - Fotografias das placas de crescimento micelial do fungo Fusarium oxysporum f.sp.cubense, em meio BDA, na concentração de $100 \mathrm{mg} / \mathrm{kg}$, na presença do complexo $\mathrm{S}$, nos períodos de 2, 4 e 7 dias.

Os complexos R e S exerceram inibição do crescimento do fungo, com redução de até $80 \%$ do diametro micelial. Nas FIGURAS 3, 4, 5 e 6, observa-se que os grânulos caóticamente dispersos no meio de cultura efetivamente inibem o crescimento micelial do fungo, pois o crescimento é inibido onde se têm os grânulos dos compostos, ocorrendo crescimento nas regiões onde não se encontram os grânulos.

\section{CONCLUSÕES}

Os complexos formados pela reação do dicloreto de difenilestanho com o ácido R-(-)-mandélico e o ácido $\mathrm{S}$-(+)-mandélico, respectivamente complexo $\mathrm{R}$ e complexo $\mathrm{S}$, foram sintetizados e caracterizados por análise elementar de carbono e hidrogênio e por espectroscopia vibracional na região do infravermelho. A análise elementar sugere uma relação estequiométrica de 1:1 entre o ácido mandélico e o dicloreto de difenilestanho. Espectros de absorção na região do infravermelho apresentaram deslocamentos da banda de estiramento $v_{\mathrm{OH}}$ do grupo alcoólico e estiramento assimétrico, $v_{\mathrm{COOH} \text { (assim.) }}$ do grupo carboxílico, para regiões de mais baixas frequiências, indicando uma coordenação por meio dos átomos de oxigênio desses grupos.

Os ácidos R-(-)- e S-(+)-mandélico mostraram-se não seletivos e com baixo poder inibidor do crescimento micelial do fungo Fusarium oxysporum f.sp.cubense. $\mathrm{O}$ dicloreto de difenilestanho mostrou-se ser inibidor do crescimento do Fusarium oxysporum f.sp.cubense. Por ser insolúvel no meio de cultura BDA, não foi possível determinar a sensibilidade do fungo em função da con- 
centração. Os complexos R e S apresentaram efetiva ação inibidora, no crescimento micelial do fungo Fusarium oxysporum f.sp.cubense; os granulos caoticamente dispersos no meio de cultura atuaram inibindo o crescimento do fungo. Não foi determinada a seletividade em termos de concentrações $(\mathrm{mg} / \mathrm{kg})$, pois os complexos são insolúveis no meio de cultura (BDA).

Com esses resultados pode-se proceder a testes in vivo com as mesmas substâncias, bem como a outros estudos sobre identificação das estruturas por RMN, CG-MS, UV em equipamentos que permitam essa determinação. Por meio desses resultados, pode-se demonstrar que essas substâncias poderão no futuro serem utilizadas no controle de doenças provocadas por esse fungo patógeno, tais como a doença murcha-dabananeira.

\section{REFERÊNCIAS BIBLIOGRÁFICAS}

BARBIÉRI, R. S.; ROCHA, J. C.; TERRA, V. R.; MARQUES NETTO, A. Método simplificado para determinação gravimétrica de zircônio ou de háfnio com ácidos $\alpha$-hidrocarboxílicos. Eclética Química, Marília, v. 14, p. 101-106, 1989.

BARBIÉRI, R. S.; TERRA, V. R.; MARQUES NETTO, A.; ROCHA, J. C. Infrared study of zirconium and hafnium $\alpha$-hydroxycarboxylates. Eclética Química, Marília, v. 19, p. 129-135, 1994.

BONNETT, R. Photosensitizers of the porphyrin and phthalocyanine series for photodynamic therapy. Chemical Society Reviews, Cambridge, v. 24, n. 1, p. 19-33, Feb. 1995.

DIAS, A. K. C. Efeito biocida de alguns $\alpha$ hidroxicarboxílicos e compostos organoestânicos sobre Fusarium oxysporum f.sp. cubense e Phytophthora capsici. 1999. 54 p. Dissertação (Mestrado em Agroquímica e Agrobioquímica) - Universidade Federal de Lavras, Lavras, 1999.

KERK, G. J. M. van der; LUIJTEN, J. G. A. Organotin compounds III: biocidal properties of organotin compounds. Journal of Applied Chemistry, Saint Paul, v. 4, n. 4, p. 314-321, Apr. 1954.
KERK, G. J. M. van der; LUIJTEN, J. G. A.; EGMOND, J. C. van; NOLTES, J. G. Progress in organotin chemistry. Chimia, Zurich, v. 16, n. 1, p. 3642, Jan. 1962.

KUMARI, A.; SINGH, I.; TANDON, J. P. Coordination behavior and microbial studies of organotin (IV) complexes of biologically active heterocyclic benzothiazolines. Main Group Metal Chemistry, Weinheim, v. 17, n. 5, p. 347-361, May 1994.

LUIJTEN, J. G. A. Applications and biologicals effects of organotin compounds. In: SAWYER, A. Organotin compounds. New York: Marcel Dekker, 1972. v. 3, p. 921-974.

MATSUGI, M.; FUKUDA, N.; MUGURUMA, Y.; YAMAGUCHI, T.; MINAMIKAWA, J.; OTSUKA, S. Catalytic asymmetric oxidation of sulfide with titaniummandelic acid complex: practial synthesis of (S)-3-[1(2-methylphenyl) imidazol-2-ylsulfinyl]propan-1-ol, the key intermediate of OPC-29030. Tetrahedron Letters, Oxford, v. 57, n. 14, p. 2739-2744, Apr. 2001.

PETTINARI, C.; MARCHETTI, F.; PETTINARI, R.; MARTINI, D.; DROZDOV, A.; TROYANOV, S. Synthesis and characterisation of tin(IV) and organotin (IV) derivatives 2-\{[(2-hydroxyphenyl) imino]methyl $\}$ phenol. Inorganica Chimica Acta, [S.1.], v. 325, n. 1/2, p. 103-114, Dec. 2001.

TERRA, V. R. Compostos organoestânicos com ácidos $\alpha$-hidroxicarboxílicos e $\alpha$-aminoácidos. 1997. 109 f. Tese (Doutorado em Química Inorgânica) - Universidade Federal de Minas Gerais, Belo Horizonte, 1997.

TERRA, V. R.; BARBIÉRI, R. S.; CASTELO BRANCO, P. A.; ABRAS, A. Síntese e caracterização de compostos diorganoestânicos com ácido dl-mandélico. Eclética Química, Marília, v. 23, p. 1730, 1998.

ZONTA, E. P.; MACHADO, A. A. Manual do Sanest: sistema de análises estatística para microcomputadores. Pelotas: UFPel, 1991. 102 p. 